\title{
Revista Colombiana de

\section{Caminata de seis minutos: propuesta de estandarización del protocolo y aplicación práctica para la evaluación de la hipertensión pulmonar con especial referencia a la de los niños}

\author{
Óscar Barón ${ }^{\mathrm{a}}$ y Gabriel Díaz ${ }^{\mathrm{b}, *}$ \\ a Departamento de Pediatría, Universidad del Rosario y Universidad de La Sabana, Fundación Neumológica Colombiana, \\ Bogotá, Colombia \\ b Departamento de Pediatría, Universidad Nacional de Colombia, Fundación Santa Fe de Bogotá, Bogotá, Colombia
}

Recibido el 26 de agosto de 2014; aceptado el 21 de mayo de 2015

Disponible en Internet el 24 de octubre de 2015

\author{
PALABRAS CLAVE \\ Ejercicio; \\ Hipertensión arterial \\ pulmonar; \\ Pediatría
}

\begin{abstract}
Resumen
Objetivo: La práctica clínica diaria exige una valoración objetiva, confiable y sencilla del paciente. En particular, en las patologías que afectan el rendimiento y la actividad física, como es el caso de la hipertensión pulmonar, es necesario conocer la repercusión que estas enfermedades tienen sobre este aspecto, básico de la vida del paciente, es decir cómo se afecta su tolerancia al ejercicio, y conocer cuál es la respuesta funcional del individuo frente a las intervenciones médicas.

Metodología: La caminata de seis minutos fue concebida como una prueba que, aunque no conlleva un esfuerzo grande para el paciente, puede cuantificar de manera bastante precisa la capacidad de tolerancia a la actividad física y sus variaciones en relación con las intervenciones terapéuticas realizadas y respecto a la propia evolución de la enfermedad.

Resultados: Una ventaja logística de la prueba es que no requiere equipos costosos, el espacio físico que se necesita es fácil de adecuar, el personal que la lleva a cabo precisa entrenamiento básico, y su interpretación es sencilla y se obtienen resultados muy confiables. Todo lo anterior, sumado a que puede repetirse con facilidad y es económica, la convierten en una prueba altamente recomendable para la evaluación y el seguimiento de pacientes pediátricos con patología cardiovascular, ya que es posible practicarla desde edades tempranas, con colaboración mínima por parte del niño evaluado.
\end{abstract}

\footnotetext{
* Autor para correspondencia.

Correos electrónicos: gfdiazg50@gmail.com, gfdiazg@unal.edu.co (G. Díaz).
} 
Conclusión: En cuanto a la hipertensión pulmonar en la edad pediátrica, es imprescindible medir de manera objetiva, sencilla y confiable la evolución del paciente, dadas las pocas opciones posibles en lo que concierne a las peculiaridades fisiológicas de los niños. De ahí que esta prueba puede constituir un punto de apoyo importante para la toma de decisiones y la evaluación del impacto de las intervenciones terapéuticas, tanto en el estado general como en la salud cardiovascular del paciente.

(C) 2015 Sociedad Colombiana de Cardiología y Cirugía Cardiovascular. Publicado por Elsevier España, S.L.U. Este es un artículo Open Access bajo la licencia CC BY-NC-ND (http://creativecommons.org/licenses/by-nc-nd/4.0/).

\section{KEYWORDS}

Exercise;

Pulmonary arterial hypertension; Paediatrics

\section{Six-minute walk test: protocol and practical application for the evaluation of pulmonary hypertension, with special reference to children}

\begin{abstract}
Objective: Clinical practice demands an objective, reliable and simple evaluation of our patients. Particularly in diseases that affect performance and physical activities, such as pulmonary hypertension, it is necessary to know the repercussions on a basic aspect in the life of the patient, that is, the tolerance to physical activity, and to know the functional response of patients to medical interventions.

Methodology: Although it does not involve great effort for the patient, the Six-Minute Walk test can provide a quantification of the capacity of the tolerance to physical activity, the response to therapeutic interventions, and patient progress.

Results: This test has logistical advantages, namely it does not require expensive devices, the physical area used is easy to prepare, the patients performing the test only need basic training and results are objective and of simple interpretation. The previous aspects and the fact that this test is easy to repeat, is inexpensive, and has no risk for the patient, makes it a highly recommended test for the evaluation and monitoring of paediatric patients with cardiovascular diseases, since it can be performed with young children because it requires minimal cooperation from them.

Conclusion: In pulmonary hypertension in children it is important to evaluate in an objective, simple and reliable way how our patients evolve, taking into account that there are few possibilities to study the physiological peculiarities of children. This test is important for decision-making and for evaluating the impact of therapeutic interventions, both from the general condition as well as from the cardiovascular health of the patient.

(C) 2015 Sociedad Colombiana de Cardiología y Cirugía Cardiovascular. Published by Elsevier España, S.L.U. This is an open access article under the CC BY-NC-ND license (http://creativecommons.org/licenses/by-nc-nd/4.0/).
\end{abstract}

\section{Introducción}

En la mayoría de las patologías, un objetivo importante en la evaluación de un paciente es la valoración del compromiso de la funcionalidad; por tanto es ideal tener al alcance alguna prueba que permita lograr este objetivo y que además ayude en el seguimiento de la evolución clínica del enfermo.

La caminata de seis minutos (C6 M) se considera como una prueba submáxima, que evalúa la capacidad para realizar actividad física semejante a la actividad de la vida diaria. Es útil para evaluar la capacidad física en enfermedades crónicas, dentro de las cuales figuran la hipertensión pulmonar ${ }^{1,2}$ y es además, la única prueba avalada por la Administración de Drogas y Alimentos, en el estudio de pacientes con hipertensión pulmonar para valorar la repercusión hemodinámica y la respuesta al tratamiento ${ }^{3}$.
En adultos existe suficiente experiencia con esta prueba, pero en los últimos años también se ha ido adquiriendo práctica en niños e incluso ya se han publicado valores normales en la edad pediátrica y en etapas cada vez más tempranas.

A continuación se presenta un protocolo, lo más completo posible, y si bien daría la impresión de ser un diseño orientado a los adultos, es completamente aplicable a cualquier edad, particularmente desde la escolar.

El objetivo fue revisar brevemente las bases fisiológicas de las pruebas de tolerancia al ejercicio, en particular la C6M, así como conocer sus indicaciones, establecer la logística necesaria para su realización, proponer un protocolo estándar, ofrecer una guía básica para la interpretación adecuada y definir su aplicación práctica en niños con patología cardiopulmonar o hipertensión pulmonar. 
Tabla 1 Pruebas de campo más utilizadas, organizadas en orden de complejidad

\section{Stair climbing test}

Caminata de 6 minutos

Test de Shuttle-walk

Test de estrés cardiaco (ergometría, protocolo de Bruce)

Test de ejercicio cardiopulmonar (ergoespirometría)

Normativa SEPAR, pruebas de ejercicio cardiopulmonar. Arch Bronconeumol. 2001.

\section{Bases fisiológicas de la evaluación del ejercicio}

La actividad física es fundamental para el desempeño de todo ser humano. Durante el ejercicio se requiere activar el metabolismo aeróbico a nivel mitocondrial, que a su vez es dependiente del aporte de oxígeno suministrado a las células desde el medio ambiente. Durante la actividad física se incrementa el funcionamiento de los sistemas cardiovascular y respiratorio en repuesta a las demandas elevadas de oxígeno, aumenta la ventilación, la perfusión alveolar, el intercambio gaseoso, el gasto cardiaco y la microcirculación periférica, en tanto que en el sistema musculoesquelético, el oxígeno es utilizado para la generación de moléculas de energía en el ciclo de Krebs. Estos mecanismos funcionan armónicamente en condiciones de salud pero puedan verse alterados en múltiples enfermedades crónicas, las cuales afectan el rendimiento del paciente y limitan su actividad física y capacidad funcional.

En las enfermedades cardiopulmonares crónicas incluyendo la hipertensión pulmonar, es importante medir el nivel de limitación funcional de los pacientes, saber su grado de limitación, así como evaluar la respuesta a las intervenciones. Para ello se han validado múltiples métodos, buscando la técnica más precisa, pero que a su vez sea más sencilla, económica, confiable y reproducible.

\section{Pruebas de campo}

A partir de la necesidad de medir la capacidad funcional del individuo frente a la actividad física se han diseñado múltiples pruebas que miden los efectos de la actividad física en circunstancias que simulan la vida diaria en espacios abiertos. Estas han variado con el tiempo, evolucionando según los avances en el entendimiento del ejercicio, buscando precisión, reproducibilidad y mínimos riesgos para el paciente. Las pruebas de campo son en general pruebas submáximas que miden la capacidad del individuo para realizar ejercicio en condiciones de esfuerzo que no lleguen al umbral anaeróbico. Miden los cambios fisiológicos que suceden a nivel cardiorrespiratorio con el fin de establecer su capacidad funcional en términos cuantificables y correlacionar los resultados con el desempeño del paciente en las actividades cotidianas. Como se muestra en la tabla 1 , se han diseñado diferentes pruebas para la evaluación funcional del ejercicio, las cuales varían en términos de complejidad, precisión y logística necesaria. En la actualidad se considera que la prueba ideal es aquella que combine estos atributos además de ser simple, económica y fácil de implementar ${ }^{4}$. Sin ser perfecta, la C6 M, reúne muchos de estos criterios.

\section{Generalidades}

La C6 M, es considerada como una de las pruebas más útiles para evaluar la capacidad funcional de los pacientes con enfermedades respiratorias y/o cardiovasculares crónicas. Es una prueba sencilla, confiable, de gran validez, fácil de aplicar, rápida y de bajo costo, que no requiere tecnología compleja, aunque precisa personal entrenado, además del cumplimiento de un protocolo muy exigente para su ejecución. Permite determinar el impacto de la enfermedad en la calidad de vida porque refleja la capacidad para realizar las actividades de la vida diaria y tiene valor pronóstico principalmente en pacientes con enfermedad pulmonar obstructiva crónica, de forma independiente o cuando se asocia a índices multidimensionales como el $\mathrm{BODE}^{1}$, de ahí su utilización en la evaluación del paciente con enfermedad cardiaca y/o pulmonar ${ }^{2,3}$.

El objetivo primordial es valorar la capacidad y la tolerancia al ejercicio en una prueba de marcha de carga sostenida, limitada por tiempo, cuya variable más importante es la máxima distancia caminada en terreno plano durante seis minutos. En términos fisiológicos, la C6M sirve para evaluar la respuesta global e integral de todos los sistemas involucrados durante el ejercicio, incluyendo el sistema cardiovascular, la circulación sistémica y periférica, la sangre, las unidades neuromusculares y el metabolismo muscular.

Mide fundamentalmente los metros caminados en una superficie plana, realizando el esfuerzo que mejor se acomode a la condición física y de tolerancia al ejercicio. Sin embargo, se pueden medir otras variables como nivel de disnea, saturación de oxígeno, frecuencia cardiaca máxima, frecuencia respiratoria, presión arterial sistémica y sensación de fatiga en las extremidades.

\section{Indicaciones y contraindicaciones de la caminata de seis minutos}

\section{Indicaciones}

a) Evaluación del estado funcional y la capacidad de ejercicio en sujetos con enfermedades respiratorias y/o cardiovasculares crónicas (las más frecuentes son la enfermedad pulmonar obstructiva crónica, asma crónica, hipertensión pulmonar, falla cardiaca, fibrosis quística, enfermedades intersticiales, etc.).

b) Evaluación de la progresión del curso clínico y el pronóstico de pacientes con enfermedades respiratorias y/o cardiovasculares crónicas, como parte integral del seguimiento de pacientes en cohortes clínicas.

c) Evaluación de la respuesta a intervenciones específicas (rehabilitación pulmonar, tratamientos farmacológicos, pre- y postoperatorios de cirugías pulmonares...).

d) Evaluación de la indicación y titulación de la oxigenoterapia crónica domiciliaria o durante el ejercicio.

\section{Contraindicaciones}

Se tienen en cuenta aquellos pacientes con enfermedad cardiovascular activa, en quienes hacer ejercicio puede poner 
en riesgo sus vidas. Las siguientes son las condiciones más frecuentes:

1. Arritmias no controladas, no diagnosticadas o no controladas que causen compromiso hemodinámico. Se recomienda tomar trazado de electrocardiograma en derivación DII en pacientes con arritmia no diagnosticada para definir el riesgo/beneficio del examen.

2. Hipertensión arterial no controlada, presión arterial sistólica mayor al percentil 95 para la edad, o presión arterial diastólica mayor al percentil 95 para la edad.

3. Frecuencia cardiaca en reposo mayor al percentil 95 para la edad.

4. Hipertensión pulmonar en pacientes con episodios recientes (dos últimos meses) de síncope, lipotimia, arritmia o inestabilidad hemodinámica.

5. Antecedentes de episodios de lipotimias o síncopes no explicados en los últimos dos meses.

6. Impedimento mental (por madurez principalmente) que inhabilite la cooperación.

7. Pacientes con clínica de exacerbación de la enfermedad respiratoria crónica, como en aquellos casos en los que desee evaluarse la capacidad funcional.

8. En todos los casos en los que el evaluador tenga dudas sobre los criterios que indican o pueden contraindicar la prueba. Se debe analizar con detalle el caso y pedir, de ser necesario, otro concepto para definir si se realiza o no la prueba.

9. No presentar condiciones que limiten las actividades físicas y que puedan interferir en la interpretación de la prueba.

\section{Preparación del paciente}

La preparación es mínima, sin embargo, en el momento de la programación de la prueba, el paciente debe recibir ciertas instrucciones:

a. Ingerir una comida ligera, bien sea en la mañana o en el inicio de la tarde; no es recomendable que el paciente asista a la prueba en ayuno.

b. Respetar los horarios de todos los medicamentos que el paciente ingiere de forma habitual (incluyendo medicamentos inhalados). Si recibe broncodilatadores deberá transcurrir un mínimo de 15 minutos desde su última utilización y el inicio de la prueba. En todos los casos se registrará la hora y la dosis de la última administración.

c. No realizar ejercicio vigoroso antes de la prueba.

d. Usar vestimenta y calzado cómodos apropiados para realizar actividad física.

e. Utilizar ayudas, si las requiere, para caminar durante la prueba (bastón, caminador, etc.).

\section{Equipos necesarios para implementar la prueba}

Los requisitos técnicos son pocos, económicos y de fácil consecución; solo se requieren los siguientes elementos:

a. Hoja de consentimiento informado.

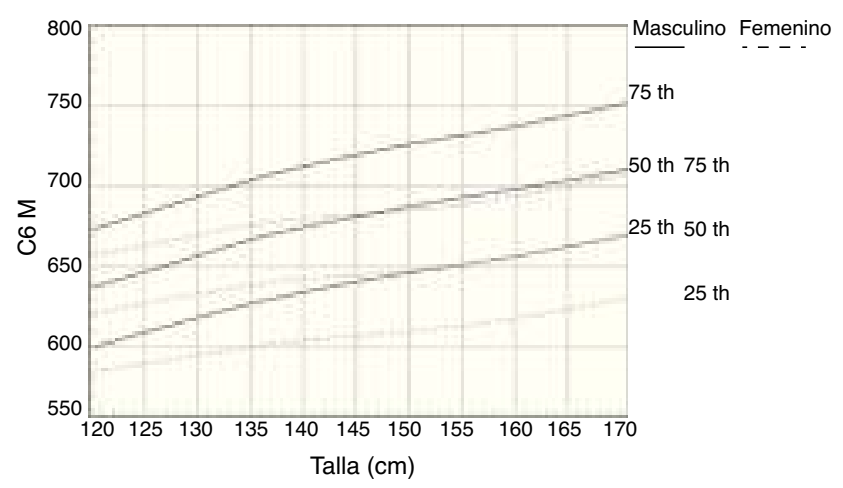

Figura 1 Distancia recorrida en TM6 según géneros.

Fuente: $L i A$, et al. Standard reference for the 6-minute walk test in healthy children aged 7 to 16 years. Am J Respir Crit Care Med. 2007.

b. Hojas preimpresas para recolección de datos (anexo 1).

c. Hoja con escalas de disnea y fatiga de Borg (adultos y modificada para niños) (anexos 2 y 3 ).

d. Cronómetro.

e. Dos conos reflectivos.

f. Metro.

g. Pulso-oxímetro.

h. Tensiómetro y fonendoscopio.

i. Sistema de oxígeno portátil (cánula y cilindro de $\mathrm{O}_{2}$ ).

j. Podómetro (opcional).

k. Sillas a ambos extremos del corredor.

l. Carro de paro con desfibrilador (cercano y disponible); en caso de un evento cardiovascular agudo, debe contarse con la posibilidad de activar un código azul.

m. Monitor de frecuencia cardiaca (opcional).

\section{Metodología}

La prueba se lleva a cabo en un corredor plano, idealmente de superficie regular, con una longitud mínima de 30 metros (figs. 1 y 2), en lo posible marcado cada metro y preferiblemente no transitado (fig. 3). Debido a que es una prueba que tiene efecto de aprendizaje, en todos los casos se efectúan dos pruebas con 30 minutos a una hora de diferencia entre una y otra, con el fin de que el paciente se familiarice; entre ambas pruebas la frecuencia cardiaca debe alcanzar un valor cercano al estado basal y deben haber desaparecido los síntomas que limitaron la primera. Las dos pruebas se realizan el mismo día para minimizar la variabilidad entre estas y evitar el efecto de entrenamiento. Podría permitirse un descanso menor a 30 minutos para iniciar la segunda prueba si el paciente ya ha recuperado los signos basales.

No se hará una segunda prueba si durante la primera caminata el paciente presenta las siguientes condiciones:

a. Disminución de la saturación de oxígeno por debajo de $80 \%$, que además se acompañe de signos y síntomas asociados a hipoxemia (inestabilidad cardiovascular, lipotimia o síncope, mareo, cambios de comportamiento, dolor precordial, disnea severa). 


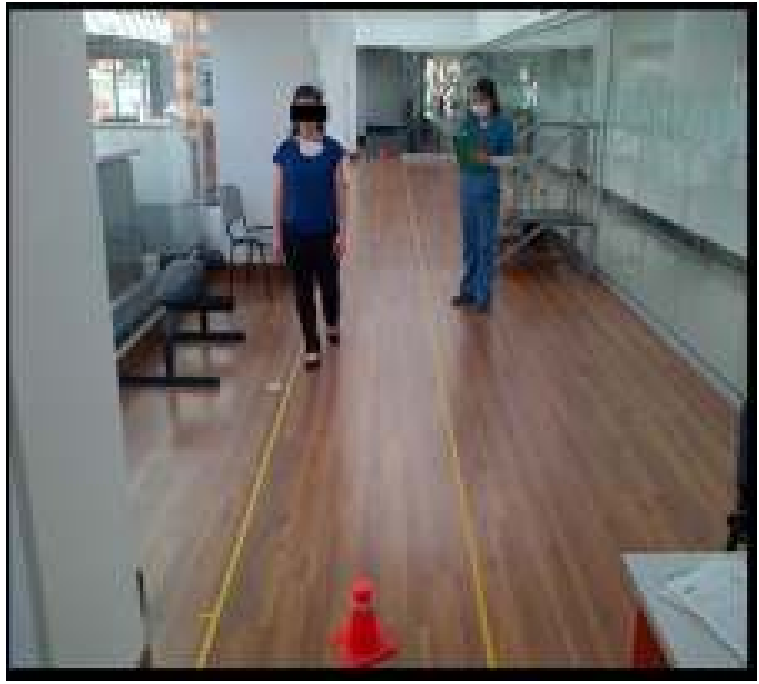

Figura 2 Ejemplo de área específica para realización de la prueba, mostrando la demarcación y conos limitantes.

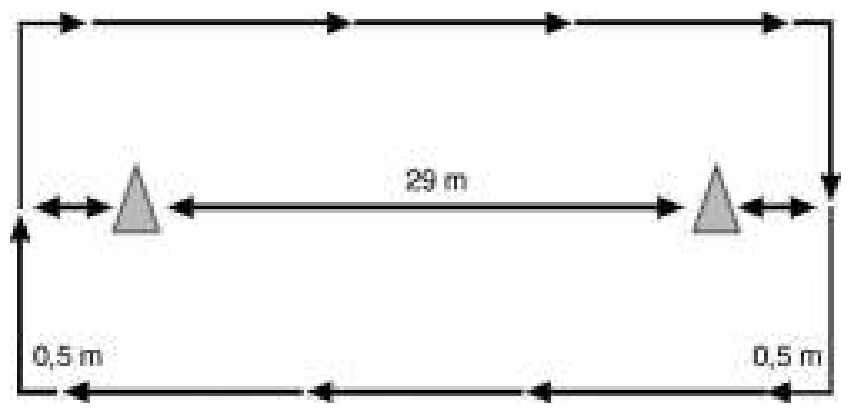

Figura 3 Recorrido durante el TM6 (los conos indican cambio de sentido).

b. Hipertensión arterial severa con el ejercicio.

c. Pulso arrítmico al finalizar la primera caminata, en paciente sin diagnóstico de arritmia previa.

d. El evaluador que realiza el examen no considera pertinente realizar una segunda prueba porque el hacerla condiciona más riesgos que beneficios.

e. El paciente no desea hacer la segunda prueba.

En el informe se reportará el motivo por el cual no se realizó una segunda caminata.

\section{Oxígeno suplementario}

Si el paciente utiliza oxígeno las 24 horas, puede realizar la prueba con el flujo de oxígeno que habitualmente usa, suministrado por bala de oxígeno portátil tipo $\mathrm{E}$. Si utiliza oxígeno menos de 24 horas, se puede realizar al aire ambiente $\left(\mathrm{FiO}_{2}\right.$ : $21 \%$ ), con el fin de conocer su capacidad funcional habitual en la vida diaria. Sin embargo, si la desaturación de oxígeno es muy marcada (saturación de oxígeno igual o inferior al $80 \%$ ), se debe realizar la caminata con oxígeno suplementario. En los casos en que el paciente tenga indicación de uso de oxígeno permanente (pese a que el paciente no lo
Tabla 2 Instrucciones preliminares

«Usted realizará la caminata de 6 minutos, cuyo objetivo es recorrer la mayor distancia posible en 6 minutos. Procure caminar tan rápido como le sea posible pero recuerde que no se trata de correr».

«Para realizar la prueba se colocarán dos conos en los extremos de un tramo de 30 metros. Usted deberá ir y volver tantas veces como le sea posible procurando no detenerse en el momento de girar alrededor de los conos en los extremos del recorrido. Durante el examen usted llevará un aparato en un dedo de la mano que permitirá conocer cómo se encuentra la oxigenación y la frecuencia del pulso durante toda la prueba». No se detenga ni disminuya la velocidad cuando me acerque a revisárselo.

«Si durante la prueba presenta síntomas intolerables que le impidan continuarla, puede disminuir la velocidad de la marcha o detenerse y si lo desea, apoyarse contra la pared o en la fisioterapeuta que le realiza la prueba. Una vez se sienta capaz de hacerlo intente proseguir la caminata; recuerde que el tiempo es limitado a 6 minutos».

«Por favor no converse ni se distraiga durante la prueba a no ser que tenga algún problema».

«La prueba comenzará con la indicación: tres, dos, uno, ¡comience! y finalizará con la indicación ipare!».

«Durante la prueba le indicaremos cada minuto el tiempo restante y al principio y al final le preguntaremos por los síntomas que presenta con esta escala (ver escala modificada de Borg) y le tomaremos la tensión arterial, el pulso y la saturación de oxígeno».

«Al finalizar la prueba usted tendrá 30 minutos o menos para descansar tras lo cual le pediremos que realice una segunda prueba con las mismas indicaciones y características de la primera».

utilizará en este horario), el evaluador puede considerar realizar la prueba con oxígeno suplementario aunque no haya disminución severa de la saturación de oxígeno en reposo (saturación de oxígeno igual o inferior al $80 \%$ ). Si se decide realizar la caminata con oxígeno suplementario el paciente permanecerá en reposo con oxígeno por lo menos durante 10 minutos. Si la prueba se hace con oxígeno suplementario, el evaluador llevará el oxígeno y caminará detrás del paciente a un metro de distancia y el oxígeno suplementario será suministrado por cilindro de oxígeno tipo $\mathrm{E}$. No deben utilizarse sistemas de oxígeno líquido, eléctricos o con flujo a demanda.

\section{Explicación preliminar para el paciente}

Es importante dar instrucciones claras de cómo se llevará a cabo la prueba antes de que esta comience. Para ello es conveniente leerle al paciente con claridad las recomendaciones que se listan en la tabla 2.

Antes de la prueba el paciente o uno de los padres firmará el consentimiento informado. 
Tabla 3 Incentivo verbal durante la prueba

Después del primer minuto, se le dice al paciente: «lo está haciendo bien, tiene cinco minutos para terminar».

Cuando faltan cuatro minutos, se le dice al paciente «está haciendo un buen trabajo, tiene cuatro minutos para terminar».

Cuando faltan tres minutos, se le dice al paciente «lo está haciendo bien, lleva la mitad del tiempo».

Cuando faltan dos minutos, se le dice al paciente «está haciendo un buen trabajo, tiene dos minutos para terminar».

Cuando falta un minuto, se le dice al paciente «lo está haciendo bien, tiene un minuto para seguir».

Quince segundos antes de terminar la prueba se le advierte que deberá detenerse cuando se le diga ipare!

Después de que el evaluador responsable informa al paciente sobre las características de la prueba, se toman los signos vitales: frecuencia cardiaca (FC), saturación de oxígeno en reposo $\left(\mathrm{SpO}_{2}\right)$, presión arterial y frecuencia respiratoria, y se registra el grado de disnea y de fatiga de las extremidades inferiores según escala de Borg ${ }^{5}$. No se permite calentamiento antes de la prueba. Estas mediciones deben realizarse con el paciente sentado durante 10 minutos antes de la prueba.

Antes de comenzar la caminata, se recordará al paciente el objetivo de la prueba «recorrer la mayor distancia posible en 6 minutos». Una vez situados (pacientefisioterapeuta) en uno de los extremos del trayecto, se dará la señal verbal de empezar a caminar (tres, dos, uno, ¡comience!) y se iniciará el cronometraje. El incentivo verbal durante la prueba se realizará cada minuto utilizando solo las frases que se enumeran en la tabla 3 con un tono estándar.

Cada minuto se registran los metros caminados (para controlar si el ritmo de la marcha es homogéneo así como la $\mathrm{FC}$ y $\mathrm{SpO}_{2}$ [ver formulario]); este es el único momento en que el fisioterapeuta podrá situarse junto al paciente. El evaluador deberá prestar especial atención en no interferir con la marcha durante la obtención de estas variables. La prueba continúa mientras la tolerancia del paciente lo permita de acuerdo con los síntomas que se observen (principalmente disnea). En ningún momento se indicará que acelere o reduzca el ritmo de la marcha.

Una vez que el paciente se ha detenido, el examinador se acercará para constatar los datos finales de la prueba: $\mathrm{SpO}_{2}$, FC, presión arterial y frecuencia respiratoria, grado de disnea y fatiga de extremidades inferiores según la escala de Borg escrita según edad del paciente (anexos 1 y 2).

Se calcula la distancia cada minuto para valorar la velocidad de la prueba, que no deben ser ni más ni menos de $0,5 \mathrm{~K} /$ hora o $0,08 \mathrm{~m} / \mathrm{s}$ a partir del tercer minuto para que la velocidad sea estable y se cumpla el precepto de prueba de carga constante.

Se realizará una segunda prueba al menos 30 minutos después. Si existe contraindicación para realizarla, se consignará en el informe el motivo de no realización.

\section{Indicaciones para suspender la prueba}

Se suspenderá la prueba si ocurre alguna de las siguientes circunstancias:

1. Negativa del paciente para continuar.

2. Decisión del evaluador o el paciente de suspender la prueba ante la presencia súbita de dolor torácico agudo o disnea intolerable, calambres musculares y/o claudicación en miembros inferiores, diaforesis inexplicada, palidez o sensación de desvanecimiento o síncope.

3. No se ha determinado un nivel de desaturación a la altura de Bogotá al cual deba suspenderse la prueba; a nivel del mar se considera un valor menor al $85 \%$. Sin embargo, el evaluador puede detener la prueba si el paciente presenta saturación de oxígeno menor al 80\% acompañada de signos y síntomas de hipoxemia severa (mareo, inestabilidad cardiovascular, lipotimia o síncope, cambios de comportamiento, dolor precordial).

4. Si por alguna razón el paciente se detiene durante la prueba, el examinador deberá asistirlo. Si este se siente capaz de continuar y el examinador no halla razón alguna para suspender la prueba, se invitará al paciente a continuar con la frase: «cuando sienta que es capaz de continuar, lo puede hacer». No se suspenderá el cronometraje mientras el paciente pare y se registrarán el número, las razones y el tiempo de las pausas que realice. En caso de que el paciente o el examinador decidan suspender la prueba, se registrará la razón de esta decisión, el tiempo transcurrido desde el inicio de la marcha y la distancia recorrida hasta ese momento.

\section{Interpretación e informe final}

El informe debe contener la siguiente información: presencia o ausencia de disnea o fatiga de miembros inferiores, $\mathrm{SpO}_{2}, \mathrm{FC}$, presión arterial, frecuencia respiratoria, distancia recorrida, tiempo ejecutado, tiempo de paradas, motivo de parada (anexo 3).

1. Describir el total de metros recorridos y expresar la distancia en porcentaje del predicho según la ecuación utilizada. Cuando el porcentaje está por encima del $80 \%$ de lo esperado o por encima del límite inferior de normalidad, se considera que el paciente caminó lo esperado.

2. Definir si el paciente alcanzó una frecuencia cardiaca máxima o submáxima.

3. Elegir la mejor de las dos pruebas (mayor distancia recorrida) y determinar el porcentaje respecto al esperado según las ecuaciones de predicción de Troosters ${ }^{3}$.

4. -Hombres $\mathrm{C} 6 \mathrm{M}=218+\left(5,14^{*}\right.$ talla $)-\left(5,32^{*}\right.$ edad $)-\left(1,8^{*}\right.$ peso $)$ $+(51,31 * 1)$.

5. - Mujeres $\mathrm{C} 6 \mathrm{M}=218+\left(5,14^{*}\right.$ talla $)-\left(5,32^{*}\right.$ edad $)-\left(1,8^{*}\right.$ peso $)$ $+\left(51,31^{*} 0\right)$.

6. En niños se puede utilizar como valor de referencia el nomograma de Li o los valores preestablecidos según la tabla de Geiger $^{6}$ (anexo 4). 
7. Se describirá el cambio en puntos de saturación de oxígeno desde el reposo hasta el sexto minuto.

8. Cuando se realiza caminata en individuos menores de cuarenta años no se reporta el porcentaje del predicho por falta de ecuación de predicción en esta edad en pacientes a la altura de Bogotá.

\section{Correlación clínica}

- En algunas situaciones clínicas, la C6 M proporciona información que puede ser un buen índice de la habilidad del paciente para realizar actividades de la vida diaria.

- La C6M tiene buena correlación con las mediciones de calidad de vida ${ }^{7}$.

- Los cambios en la C6M posterapia tienen correlación con la mejoría subjetiva de la disnea.

- Hay correlación significativa $(r=0,73)$ con el consumo de oxígeno pico en pacientes con enfermedad pulmonar avanzada $^{8}$.

- Los cuestionarios de estado funcional son más variables $(22-33 \%)$ que la $\mathrm{C} 6 \mathrm{M}^{7}$.

\section{Limitaciones}

Aunque son pocas, existen algunas que se señalan a continuación:

- No determina la captación de oxígeno máximo.

- No diagnostica la causa determinante de la disnea con el ejercicio.

- No evalúa causas o mecanismos precisos de limitación con ejercicio ${ }^{8}$.

- La información proporcionada por la C6 M debe ser considerada complementaria a la comprobación del ejercicio cardiopulmonar y no un reemplazo.

\section{Ventajas}

- En pacientes con enfermedad cardiopulmonar crónica, en particular en la hipertensión pulmonar, la C6M evalúa de manera adecuada la capacidad funcional y la tolerancia al ejercicio. Es una prueba sencilla, económica, reproducible y fácil de implementar (mínimo equipo y personal) e interpretar. Tiene, además, muy buena correlación con las actividades cotidianas y ha sido estudiada y validada, convirtiéndose en la única prueba validada por la Administración de Drogas y Alimentos para la evaluación y el seguimiento de pacientes con hipertensión pulmonar $^{4}$.

- Tiene mejor reproducibilidad que el VEF1 en enfermedad pulmonar obstructiva crónica? .

- Se asemeja al shuttle-walk test, si bien este ha sido menos validado ${ }^{4}$, menos usado y tiene mayor potencial de problemas cardiovasculares.

\section{Comentarios finales}

En adultos la C6M es útil y fácilmente aplicable; de ahí la frecuencia con que se practica este examen. Sin embargo, esta situación difiere en niños por muchos aspectos. Pese a ello, hay cada vez más estudios publicados sobre su utilidad y confiabilidad en la edad pediátrica9 ${ }^{9}$ incluso en niños muy enfermos ${ }^{10}$ aunque la mayoría de los estudios se han hecho con niños mayores de 12 años $^{11}$, hecho que dificulta su interpretación por muchas variables en niños menores de 8 años.

Es importante señalar que ya existen estudios, como el de Priesnitz ${ }^{12}$ y Lammers ${ }^{3}$ en menores de doce años. En este último se incluyeron 328 niños entre 4 y 12 años y se reportaron los valores normales en este grupo de edad, señalando que la extensión caminada aumenta de manera significativa entre los 4 y los 7 años y a partir de esta edad se estabiliza. Parecería que ya estaría resuelto el tema sobre parámetros normales para este grupo de edad; sin embargo, otros estudios señalan la dificultad de extraer parámetros normales por la diversidad de variables relacionadas, incluso con la raza, el tamaño de los niños con relación a la edad y la longitud de las piernas. Para completar las dudas, el estudio de Klepper $^{13}$ realizado con niños estadounidenses señala que los resultados de estudios hechos en niños de determinado país, no son aplicables a niños de otros países, y algo semejante pero relacionado con diferentes razas se ha publicado en adultos ${ }^{14}$. En estos estudios se valora, entre otros parámetros, la saturación y la frecuencia cardiaca. En cuanto a la dificultad y la necesidad de hacer estudios en grupos de pacientes específicos, al analizar el trabajo de Lammer et al. ${ }^{3}$ no se observa gran diferencia en la saturación con el ejercicio ya que es una prueba submáxima, con saturación alrededor del $94 \%$. Si se considera al niño habitante de altura elevada sobre el nivel del mar, en quien dada la hipoxia hipobárica se obtienen valores de saturación del $90 \%$ o un tanto por debajo, se entiende que esta es una variable a tener en cuenta y que por ende se requieren estudios específicos, e incluso a otras alturas, para determinar los parámetros normales. A esto se suma que estos grupos étnicos en general son de talla más baja y por tanto de piernas más cortas, característica que influye en los parámetros normales durante la realización de la prueba.

En vista de lo anterior, en una ciudad de base como Bogotá, situada a 2.600 msnm es compresible que se tengan parámetros propios que requieren la aplicación de protocolos con dicho fin.

\section{Responsabilidades éticas}

Protección de personas y animales. Los autores declaran que para esta investigación no se han realizado experimentos en seres humanos ni en animales.

Confidencialidad de los datos. Los autores declaran que en este artículo no aparecen datos de pacientes.

Derecho a la privacidad y consentimiento informado. Los autores declaran que en este artículo no aparecen datos de pacientes.

\section{Conflicto de intereses}

Los autores declaran no tener ningún conflicto de intereses. 


\section{Anexo 1. Escala de disnea de Borg (adultos)}

Escala de disnea de Borg (adultos)

Califique su ahogo y cansancio según la tabla:

\begin{tabular}{ll}
\hline 10 & Máximo \\
9 & Muy, muy severo \\
8 & \\
7 & Muy severo \\
6 & \\
5 & Severo \\
4 & Algo severo \\
3 & Moderado \\
2 & Leve \\
1 & Muy leve \\
0,5 & Muy, muy leve \\
0 & Nulo \\
\hline
\end{tabular}

Anexo 2. Escala de Borg modificada, para niños

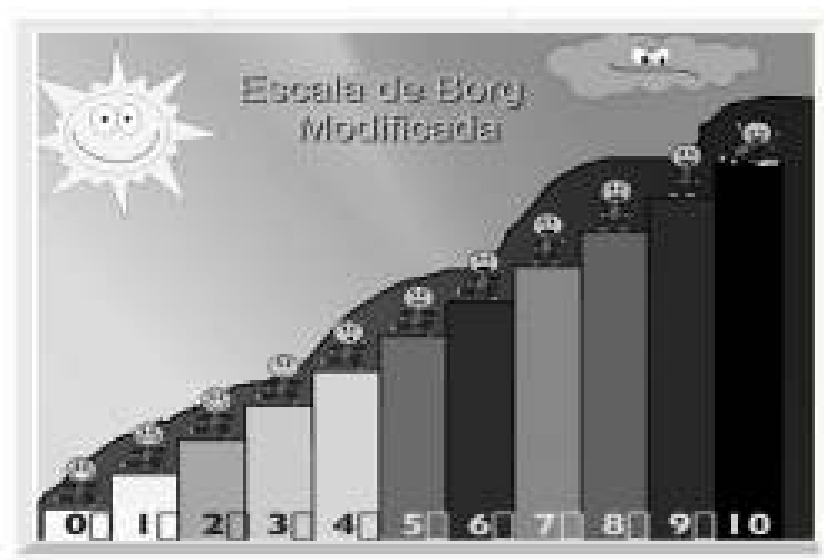

\section{Anexo 3. Formato de reporte de la C6 M}

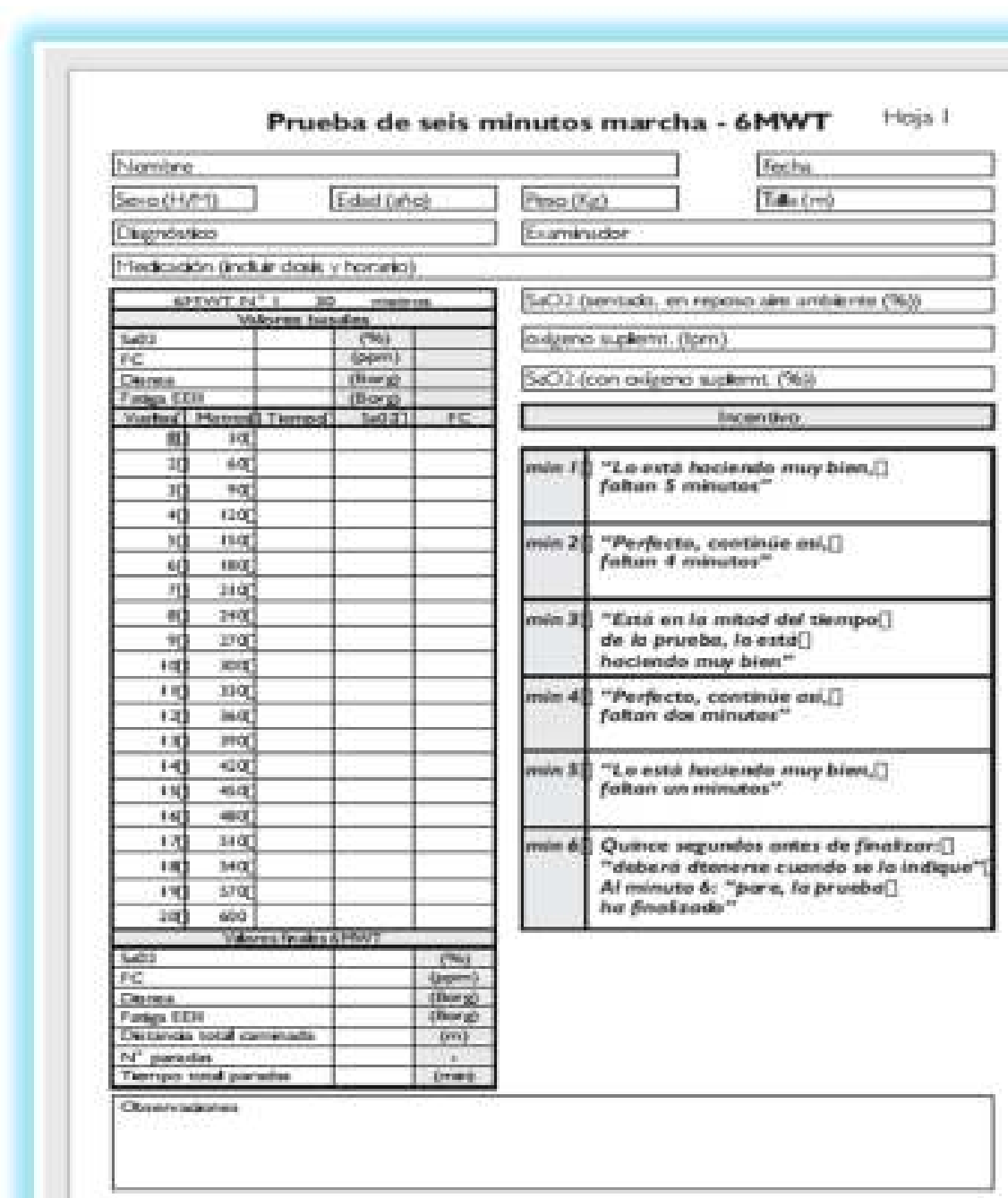




\section{Anexo 4. Valores de distancia caminada en niños sanos según edad y sexo propuesto por Geiger}

Tabla D.1.

Tabla D.1 Valores de distancia caminada en niños sanos según edad y sexo propuesto por Geiger

\begin{tabular}{|c|c|c|c|c|c|c|}
\hline Género & Edad & $\mathrm{n}$ & mediana (rango) & $95 \%$ rango & Media \pm DS & $95 \%$ IC \\
\hline \multirow[t]{5}{*}{ Hombre } & 3 a 5 y & 22 & $544.3(318.0-680.6)$ & $319.7-680.6$ & $536.5 \pm 95.6$ & 491.1-578.9 \\
\hline & 6 a $8 y$ & 66 & $584.0(455.0-692.0)$ & $471.0-659.3$ & $577.8 \pm 56.1$ & $564.0-591.6$ \\
\hline & 9 a 11 y & 57 & $667.3(540.2-828.0)$ & $556.2-801.5$ & $672.8 \pm 61.6$ & $656.5-689.2$ \\
\hline & 12 a 15 y & 80 & $701.1(276.1-861.0)$ & $600.7-805.3$ & $697.8 \pm 74.7$ & $681.2-714.4$ \\
\hline & 16 y o mayor & 55 & $727.6(569.0-865.3)$ & $616.9-838.4$ & $725.8 \pm 61.2$ & $709.3-742.4$ \\
\hline \multirow[t]{5}{*}{ Mujer } & 3 a 5 y & 25 & $492.4(352.0-713.3)$ & $364.5-692.7$ & $501.9 \pm 90.2$ & 464.7-539.1 \\
\hline & 6 a 8 y & 46 & $579.3(406.0-707.2)$ & $448.8-693.9$ & $573.2 \pm 69.2$ & $552.7-593.8$ \\
\hline & 9 a 11 y & 62 & $655.8(548.0-818.0)$ & $572.0-760.5$ & $661.9 \pm 56.7$ & $647.4-676.3$ \\
\hline & 12 a $15 y$ & 71 & $657.6(485.5-785.0)$ & $571.2-746.5$ & $663.0 \pm 50.8$ & $651.0-675.0$ \\
\hline & 16 y o mayor & 44 & $660.9(557.0-774.3)$ & $571.2-756.2$ & $664.3 \pm 49.5$ & $649.3-679.3$ \\
\hline
\end{tabular}

Fuente: Geiger R et al. ${ }^{15}$.

\section{Bibliografía}

1. Celli BR. Pulmonary rehabilitation in patients with COPD. Am J Respir Crit Care Med. 1995;152:861-4.

2. Solway S, Brooks D, Lacasse Y, Thomas S. A qualitative systematic overview of the measurement properties of functional walk tests used in the cardiorespiratory domain. Chest. 2001;119:256-70.

3. Miyamoto S, Nagaya N, Satoh T, Kyotani S, Sakamaki F, Fujita $M$, et al. Clinical correlates and prognostic significance of six-minute walk test in patients with primary pulmonary hypertension. Comparison with cardiopulmonary exercise testing. Am J Respir Crit Care Med. 2000;161:487-92.

4. Lammers AE, Hislop AA, Flynn Y, Haworth SG. The six-minute walk test: Normal values for children of 4-11 years of age. Arch Dis Child. 2008;93(6):464-8.

5. Borg GAV. Psycosocial bases of perceived exertion. Med Sci Sports Exer. 1982;14:377-81.

6. Fumagalli E, Olivera $M$, Simoes $M$, Silva I. Utilización del test de caminata de 6 minutos en el manejo de la hipertensión pulmonar en niños. Arq Bras Cardiol. 2010;95(1):e10-3.

7. Guyatt GH, Townsend M, Keller J, Sionger JM, Nogradi S. Measuring functional status in chronic lung diseases. Respire Med. 1991;85 suppl B:17-21.
8. Wasserman K, Hnaeen JE, Sue DY, Casaburi R, Whipp BJ. Principles of exercise testing and interpretation. $3{ }^{\text {rd }}$. ed. Philadelphia: Lippincott, Williams \& Wilkins; 1999.

9. Butland TJA, Pang J, Gross ER. Two, six, and 12-minute walking test in respiratory disease. BMJ. 1982;284:1607-8.

10. Li AM, Yin J, YU CC, Tsang T, Wong E, Chan D, et al. The six minute walk test in healthy children: reliability and validity. Eu Respir J. 2013;25(6):1057-60.

11. Nixon P, Joswiak ML, Fricker FJ. A six-minute walk test for assessing exercise tolerance in severely ill children. J Pediatr. 1996;129:362-6.

12. Hassan J, van der Net J, Helders PJM, Prakken BJ, Takken T. Six-minute walk test in children with chronic conditions. Br J Sports Med. 2010;44:270-4.

13. Priesnitz CV, Rodrigues GH, da Silva G, Stumpf C, Viapiana G, Cabral CP. Reference values for the 6-min walk test in children who are healthy aged 6-12 years. Pediatr Pulmonol. 2009;44:1174-9.

14. Klepper S, Muir N. Reference values on the 6-minute walk test for children living in the united states. Pediatric Physical Therapy. 2011;23(1):32-40.

15. Geiger R, Strasak A, Treml B, Gasser K, Kleinasser A, Fischer $\mathrm{V}$, et al. Six minute walk test in children and adolescents. J Pediatrics. 2007;150:395-9. 